\title{
Numerical Simulation of a Laboratory-Scale Turbulent Slot Flame
}

\author{
John B. Bell ${ }^{\mathrm{a}}$, Marcus S. Day ${ }^{\mathrm{a}}$, Joseph F. Grcar ${ }^{\mathrm{a}}$ \\ Michael J. Lijewski ${ }^{\mathrm{a}}$, James F. Driscoll ${ }^{\mathrm{b}}$, Sergei A. Filatyev ${ }^{\mathrm{c}}$ \\ ${ }^{a}$ Computational Research Division, Lawrence Berkeley National Laboratory, Berkeley, CA 94720, USA \\ b Department of Aerospace Engineering, University of Michigan, Ann Arbor, MI 48109, USA \\ ${ }^{\mathrm{c}}$ Department of Mechanical Engineering, Purdue University, West Lafayette, IN 47907-2088. USA
}

\begin{abstract}
We present three-dimensional, time-dependent simulations of the flowfield of a laboratory-scale slot burner. The simulations are performed using an adaptive time-dependent low Mach number combustion algorithm based on a second-order projection formulation that conserves both species mass and total enthalpy. The methodology incorporates detailed chemical kinetics and a mixture model for differential species diffusion. Methane chemistry and transport are modeled using the DRM-19 mechanism along with its associated thermodynamics and transport databases. Adaptive mesh refinement dynamically resolves the flame and turbulent structures. Detailed comparisons with experimental measurements show that the computational results provide a good prediction of the flame height, the shape of the time-averaged parabolic flame surface area, and the global consumption speed (the volume per second of reactants consumed divided by the area of the time-averaged flame). The thickness of the computed flame brush increases in the streamwise direction, and the flame surface density profiles display the same general shapes as the experiment. The structure of the simulated flame also matches the experiment; reaction layers are thin (typically thinner than $1 \mathrm{~mm}$ ) and the wavelengths of large wrinkles are $5-10 \mathrm{~mm}$. Wrinkles amplify to become long fingers of reactants which burn through at a neck region, forming isolated pockets of reactants. Thus both the simulated flame and the experiment are in the "corrugated flamelet regime."
\end{abstract}

Keywords: turbulent premixed combustion, low Mach number flow, adaptive mesh refinement

\section{Introduction}

Premixed turbulent flames are of increasing practical importance and remain a significant research challenge in the combustion community. To investigate experimentally the interaction of turbulence with the flame front, a variety of simplified flame configurations have been studied which can be catego- 
rized by the flame stabilization mechanism. Recent examples include studies by Sattler et al. [1] of a turbulent V-flame, Shepherd et al. [2] of a swirlstabilized flame, Most et al. [3] of a bluff-body stabilized flame, Chen et al. [4] of Bunsen and stagnation flames. Modern experimental diagnostics as well as theory (see, for example, Peters [5]) have made substantial progress in understanding basic flame physics for these idealized laboratory flames and developing models that can be used for engineering design. However, recent development in numerical methodologies for simulating reaction flows coupled to modern high-performance computer architectures have made it possible to simulate these types of laboratory-scale flames without needing to introduce closure models for turbulence or turbulent chemistry interaction. See Vervisch et al. [6] and Bell et al. [7] for simulations of turbulent V-flames.

Here we consider the slot Bunsen flame investigated by Filatyev et al. [8]. The sequence of experiments introduced in their work were designed to be well-suited for numerical simulation. Specifically, the burner consists of a simple slot flanked by pilots to anchor the flame and shield it from the laboratory environment. In this paper, we present simulation results for one of the experimental cases. Several comparisons are made between the simulation and the experiments.

\section{Experimental setup}

The slot burner experiment, which is described in Filatyev et al. [8], consists of three rectangular burners: a central burner and two side burners. Each of the three rectangular burners had a cross section of $2.5 \mathrm{~cm} \times 5 \mathrm{~cm}$ and a length of $20 \mathrm{~cm}$. Stoichiometric methane-air mixtures were provided to each burner. The central burner was used to create the Bunsen flame of interest while the side burners contained flat flames that provided hot products at a velocity that is matched to the velocity of products from the Bunsen flame. The side burners eliminated any shear layers that can occur at the boundary where products meet the room air; these shear layers can produce unwanted turbulence. Significantly, the only experimentally reported parameters available to characterize the incident turbulence field were the r.m.s. velocity fluctuations and the streamwise integral length scale. Although several flame conditions were achieved in the experiment, only one (case $3 b$ ) was numerically simulated. This case corresponds to a mean velocity of 3 $\mathrm{m} / \mathrm{s}$ and a turbulence intensity of $10 \%$ with an integral scale $\ell_{t}=5.2 \mathrm{~mm}$.

The slot burner flame represents one of the major categories of turbulent premixed combustion: it is an "envelope flame" [9] that has a base that is anchored; all of the reactants must flow directly toward the flame (without large streamline bending) and this leads to unusually large stretch rates [8]. Mean properties are $2 \mathrm{D}$, which reduces inaccuracies associated with using 2D light sheet measurements to characterize 3D flame properties. Other general configurations of turbulent premixed flames include spherically-expanding flames, V-flames, and counterflow or low-swirl flames.

The flame height is defined as the height on the centerline of the contour associated with a mean reactedness $\bar{c}$ of 0.5 . Reactedness was measured in the experiment from Mie-scattering images created using oil droplets seeded in the reactant stream. The turbulent flame brush thickness $\left(d_{T}\right)$ was defined as the full width at half maximum of the profiles of the r.m.s. fluctuations in reactedness $\left(c_{\mathrm{rms}}^{\prime}\right)$. Particle image velocimetry and laser velocimetry were used to measure velocity fluctuations and the streamwise integral scale of the incident turbulence. Flame surface density was measured using images of $\mathrm{CH}$ fluorescence obtained by tuning the $390 \mathrm{~nm}$ sheet from a Nd:YAG pumped dye laser to an absorption line of $\mathrm{CH}$. The flame front curvature also was measured. Procedures for extracting the latter two quantities from the experimental database are discussed in Reference [8].

\section{Computational model}

The simulation is based on a low-Mach-number formulation in which the background thermodynamic pressure remains constant. The methodology treats the fluid as a mixture of perfect gases, and uses a mixture-averaged model for differential species diffusion, ignoring Soret, Dufour and radiation effects. With these assumptions, the equations governing the flow are given by

$$
\begin{aligned}
\frac{\partial \rho U}{\partial t}+\nabla \cdot \rho U U & =-\nabla \pi+\nabla \cdot \tau+\rho g \hat{z} \\
\frac{\partial \rho Y_{m}}{\partial t}+\nabla \cdot U \rho Y_{m} & =\nabla \cdot \rho D_{m} \nabla Y_{m}-\dot{\omega}_{m} \\
\frac{\partial \rho h}{\partial t} & +\nabla \cdot U \rho h=\nabla \cdot \frac{\lambda}{c_{p}} \nabla h+ \\
& +\sum_{m} \nabla \cdot h_{m}\left(\rho D_{m}-\frac{\lambda}{c_{p}}\right) \nabla Y_{m}
\end{aligned}
$$

where $\rho$ is the density, $U$ is the velocity, $Y_{m}$ is the mass fraction of species $m, h$ is the mass-weighted enthalpy of the gas mixture, $T$ is the temperature, and $\dot{\omega}_{m}$ is the net destruction rate for specie $m$ due to chemical reactions. Also, $\lambda$ is the thermal conductivity, $\tau$ is the stress tensor, $c_{p}$ is the specific heat of the mixture, $h_{m}(T)$ and $D_{m}$ are the enthalpy and species mixture-averaged diffusion coefficients of species $m$, respectively, $g$ is the gravitational acceleration, and $\hat{z}$ is oriented downward. These evolution equations are supplemented by an equation of state for a perfect gas mixture:

$$
p_{0}=\rho R_{m i x} T=\rho \mathcal{R} T \sum_{m} \frac{Y_{m}}{W_{m}}
$$

where $W_{m}$ is the molecular weight of species $m$, and $\mathcal{R}$ is the universal gas constant. In the low Mach num- 
ber model, the equation of state constrains the evolution and removes acoustic wave propagation from the dynamics of the system.

Our basic discretization algorithm combines a symmetric operator-split treatment of chemistry and diffusion processes with a density-weighted approximate projection method for incorporating the velocity divergence constraint arising from the low-Machnumber formulation. This basic integration scheme is embedded in a parallel adaptive mesh refinement (AMR) algorithm based on on a block-structured hierarchical grid system composed of nested rectangular grid patches $[10,11]$. The adaptive algorithm is second-order accurate in space and time, and discretely conserves species mass and enthalpy. No explicit model for turbulence or turbulence / chemistry interaction is included. The reader is referred to [12] for details of the low-Mach-number model and its numerical implementation and to [7, 13] for previous applications of this methodology to the simulation of premixed turbulent flames.

The simulation domain is a volume of $375 \mathrm{~cm}^{3}$ that begins at the burner exit: $7.5 \mathrm{~cm}$ wide $(-3.75 \leq$ $x \leq 3.75), 5 \mathrm{~cm}$ deep $(0 \leq y \leq 5)$ and $10 \mathrm{~cm} \mathrm{high}$ $(0 \leq z \leq 10)$. As in the experiment, the two pilot flames, each $2.5 \mathrm{~cm} \times 5 \mathrm{~cm}$, flank the central turbulent burner $(2.5 \mathrm{~cm} \times 5 \mathrm{~cm})$ across the width of the simulation domain. At $y=0$ and 5 we have assumed periodic boundary conditions. For the simulation, at $x= \pm 3.75$ and at $z=10$ we have imposed outflow boundary conditions. At the inflow face we approximate the pilot flames by a uniform inflow of hot combustion products at a velocity of $7 \mathrm{~m} / \mathrm{sec}$. For the central burner we impose a uniform mean inflow of 3 $\mathrm{m} / \mathrm{sec}$ with superimposed turbulent fluctuations. The inflow composition is a methane-air mixture at equivalence ratio of one and $T=298$. The turbulent fluctuations are generated in a separate computation by evolving a random initial condition with an incompressible Navier Stokes solver. This evolution guarantees that we match the experimental turbulent intensity and integral length scale, and that the phase properties of the fluctuations are realistic. The simulations were done with an initial base grid of $120 \times 80 \times 160$ with two additional levels of refinement for an effective resolution of $480 \times 320 \times 640$. The refinement criteria were selected so that the cold reactants entering the central burner and the flame zone were always modeled at the highest resolution. In addition, strong vortical structures in the post flame zone were refined.

The chemical kinetics are modeled using the DRM-19 subset of the GRI-Mech 1.2 methane mechanism [14]. DRM-19 is a detailed mechanism containing 20 chemical species and 84 fundamental reactions. Transport and thermodynamic properties are from the GRI-1.2 databases.

A detailed convergence analysis for the method in general is presented in the cited references. The following additional tests were used to validate the resolution for this particular case: (1) a well-resolved simulation in the same configuration was carried out using a simpler two-step methane mechanism, and in terms of the statistics presented in the paper, was in good agreement, both with the experimental data and with those of the more detailed 84-step chemical mechanism presented; (2) a flat-flame simulation using the temporal and spatial resolution of the present study recovers a burning speed consistent with published experimental data (and the two-step test mechanism); (3) limited tests at higher spatial resolution show no substantive change in flame or turbulence propagation dynamics, although these higherresolution runs were too expensive to generate the statistical quantities presented in the manuscript. We also note that at $1 / 2$ of the Kolmogorov length, the mesh spacing used for the flow upstream of the flame is more than adequate to resolve the turbulence. See also, Bell et al. [7] for a discussion of resolution issues in a similar context.

\section{Results}

\subsection{General Overview of the Flame}

A 3D view of the instantaneous flame surface as predicted by the simulation appears in Fig. 1. The premixture issuing from the central slot ignites on contact with the hot, stabilizing coflows to form two flame sheets anchored at each lip of the slot. Above $1 \mathrm{~cm}$, the flame sheets exhibit the crenelated (cusped) texture characteristic of premixed flames in mild turbulence. In particular, this image shows the sharp ridges of high negative curvature surrounding large pockets of modest positive curvature. Here, curvature is defined such that positive curvature is convex with respect to reactants. (For similar features in a rod stabilized flame, see [7].)

The thin ridges of large negative curvature are the dominant feature of the flame as the two flame sheets merge at the flame tip. The elevation where the two sheets merge changes with the shape of the ridges and fluctuates rapidly. For example, in Fig. 2, the flame height varies from 4 to $6 \mathrm{~cm}$ along the slot. The thin ridges may burn through to detach portions of the flame surface. In Fig. 1, an island has been nearly completely consumed while two more are about to form.

Figure 2 shows detail of the chemical structure of the flame. In Fig. 2d, the blue color that surrounds the white, unburned region indicates that molecular hydrogen diffuses to the unburned side of the flame - as expected in a premixed methane flame. The concentration of hydrogen can be comparatively high through the entire thickness of a thin ridge. Trace amounts hydrogen increase methane flame speed and resistance to extinction from stretching, so the presence of hydrogen may account for the resilience of the ridges once they form. On the burned side, Figs. 2c-e show that the major products and heartier intermediates remain out of equilibrium some distance from the flame sheet. Since the oxidation of carbon monoxide releases a large fraction of the heat, the temperature 
(not shown) also remains below its final value some distance from the flame sheet. The main $\mathrm{C}_{1}$ reaction path for this flame is $\mathrm{CH}_{4} \rightarrow \mathrm{CH}_{3} \rightarrow \mathrm{CH}_{2} \mathrm{O} \rightarrow$ $\mathrm{HCO} \rightarrow \mathrm{CO} \rightarrow \mathrm{CO}_{2}$. Fig. $2 \mathrm{~b}$ shows that the peak concentrations of intermediates such as $\mathrm{CH}_{2} \mathrm{O}$ may vary along the flame sheet. Figure $2 \mathrm{f}$ shows a planar laser-induced fluorescence (PLIF) experimental image of the $\mathrm{CH}$ profile. The experimental image shows qualitatively similar features to the simulation data. We note that the size of the PLIF window makes it impossible to image the entire flame so the experimental profile is a composite of two separate images.

The velocity structure of the flow is depicted in Fig. 3 , which shows the magnitude of vorticity through a slice of the flame. The figure shows essentially isotropic vorticity entering the domain. In the postflame zone the vorticity is dominated by baroclinic vorticity generated in the regions of high negative curvature as evidenced by the intense regions found on each side of the sharp cusps in the flame surface. Vortical structures passing through the flame are reduced in magnitude by the expansion of the fluid and play little role in the post-flame velocity structure. The vorticity depicted in Fig. 3 is consistent with the baroclinic vorticity measured by Mueller et al. [15].

\subsection{Flame Brush}

Figure 4 shows good agreement between flame brushes calculated from the experiment and the simulation. Experimental values are obtained from Mie scattering images of oil droplets in the unburned mixture. Signals from 73 images are averaged after each is normalized to the interval $0-1$. Simulation values are from a similar reduction of temperature, using the $650 \mathrm{~K}$ droplet vaporization as a cutoff between 0 and 1 , then integrating the 3D field along the length of the slot and averaging over 88 snapshots, separated in time by $125 \mu \mathrm{s}$. The experiment and the simulation both have an average flame height of approximately $4 \mathrm{~cm}$. Measuring the length of the $\bar{c}=0.5$ contour from the simulation data, we can define a global turbulent flame speed as the average consumption speed over this area required to consume the fuel. This gives a result of $2.45 \times s_{L}$ compared to $2.55 \times s_{L}$ for the experimental data. (See [8] for details of how the turbulent flame speed is computed for the experimental data.) The width of the flame brush (defined as the full width half maximum of $c_{r m s}^{\prime}$ ) was computed both from the experimental data, and from the binarized simulation data. Along the flanks of the flame, the two measures agreed remarkably well, increasing linearly from $2 \mathrm{~mm}$ at an elevation of $1 \mathrm{~cm}$ to approximately $11 \mathrm{~mm}$ at $3.5 \mathrm{~cm}$. Agreement in flame brush thickness profiles provides a further level of verification that the simulation is correctly capturing the fluctuating flame surface dynamics on the longer timescale associated with turbulent structure advection.

Although the simulation and experiment are quite similar, there are two notable differences. The flanks of the experimental flame are more vertical than the simulation and the thickness of the flame brush at the flame tip shows less variation than the simulation. Several factors could be responsible for these differences. A general issue in this type of simulation is that the turbulent inflow is characterized only by its intensity and integral length scale. A better match to flame details would be possible if a more complete characterization of the turbulence were available.

In the experiment the measured centerline velocity was less than the mean inflow velocity. Conservation considerations then imply that the velocity at the outer edges of the burner are somewhat higher. Since one expects the turbulent intensity to be proportional to the mean inflow velocity, this suggests reduced turbulent intensity in the center as well. The simulation, on the other hand, used a top-hat velocity profile. The two observed differences in the flame brush are consistent with these differences in inflow conditions.

\subsection{Flame Surface Density}

Figure 5a,c show the experimental flame surface densities from Filatyev et al. [8]. That data is obtained by reducing PLIF images of $\mathrm{CH}$ to pixels of 0 or 1 . Due to resolution issues in the diagnostics, only the upper portion of the experimental data is presented. The length of the $\mathrm{CH}$ surface in $2 \mathrm{~mm}$ square bins was determined by counting the pixels where $\mathrm{CH}$ is present, and multiplying by the ratio of pixel area to the average thickness of the imaged $\mathrm{CH}$ profile $(0.7$ $\mathrm{mm})$. This process was repeated and the results averaged over 100 images.

Flame surface density is computed two ways from the simulation. The $2 \mathrm{D}$ method is intended to be analogous to the processing of experimental data. At each of 88 time snapshots, cross sections of the $1684 \mathrm{~K}$ isosurface are taken every $3 \mathrm{~mm}$ along the slot, producing 1408 2D flame contours. The contours are then binned into $2 \mathrm{~mm}$ squares. The total length per bin, divided by the area of the bin and averaged over 50 flame snapshots, produces $\Sigma_{2 D}$. The calculation was repeated for $1 \mathrm{~mm}$ bins with little change in outcome; results for both bin sizes are shown in Figs. $5 \mathrm{~b}$ and e. Alternatively, the 3D method tabulates the area of the $1684 \mathrm{~K}$ isotherm that lies in bins running the length of the slot, that is, without first creating $2 \mathrm{D}$ contours. Again there was little to distinguish between bins whose cross section is 1 or $2 \mathrm{~mm}$ square; both are shown in Figs. 5c and f. Comparison of the 2D (left) and 3D (right) sides of Fig. 5 reveals that the 2D method systematically underestimates $\Sigma$ by 25 $33 \%$ through the central portion of the flame from 3-6 $\mathrm{cm}$ above the burner exit. The experimental and simulation data are similar in structure as seen in both the planar image and the cuts of the data at several heights.

\subsection{Flame Surface Curvature}

We examine the structure of the flame in greater detail by looking at its curvature. The mean curva- 
ture of a surface is $M=\left(R_{1}^{-1}+R_{2}^{-1}\right) / 2$ where $R_{1}$, $R_{2}$ are the principle radii of curvature. An equivalent formula for an isotherm is $M=(\nabla \cdot \hat{n}) / 2$ where $\hat{n}=-\nabla T /\|\nabla T\|$. Since $\nabla T \neq 0$ near the flame, we can evaluate $M$ in the simulation and map the values to the $1684 \mathrm{~K}$ isotherm that we associate with the flame sheet. In the experiment, we have only vertical slices of flame locations as indicated by Mie scattering images or PLIF data. Here, we use the binarized Mie scattering images to identify the flame surface. The interface is fit with a quartic function and the $2 \mathrm{D}$ curvature is evaluated at uniform intervals along the interface. Finally, a procedure similar to the experimental data processing was conducted using planes of the 3D simulation data.

Figure 6 shows probability density functions (PDF's) of the curvature for both the experiment and the simulation computed in 2D planes. The experimental data processed 50 Mie scattering frames. From the simulation, 16 planes at $3 \mathrm{~mm}$ intervals were extracted from each of 88 time snapshots. The distributions peak near zero and are nearly symmetric, but the distributions show the expected negative skewness associated with a propagating flame, see $[16,17]$

The red curve in Fig. 6 is a PDF of mean curvature computed from the 3D simulation data. The central region of the distribution is surprisingly similar to the $2 \mathrm{D}$ processing method: nearly symmetric with a slight skewing to the negative side. This similarity between 2D and 3D is somewhat surprising. Ashurst and Shepherd [18] suggest that 2D curvature computations should overestimate the $3 \mathrm{D}$ value. The other interesting feature of the 3D curvature PDF is the enhancement of the tail in the negative curvature compared to 2D data. We conjecture this reflects some bias in the orientation of the blue cusps in Fig. 1.

The Gaussian curvature, $G=R_{1} R_{2}$, may be computed using expressions reviewed in [19] and then combined with $M$ to find the radii of curvature, $R_{1}, R_{2}=M \pm \sqrt{M^{2}-G}$. Following Pope et al. [20], we define the shape factor, $S$

$$
S= \begin{cases}R_{1} / R_{2} & \text { if }\left|R_{1}\right|<\left|R_{2}\right| \\ R_{2} / R_{1} & \text { otherwise }\end{cases}
$$

The PDF of $S$ in Fig. 7 indicates that $S$ is strongly peaked near zero. Thus, the highest probability configuration of the flame is locally cylindrical. However, a significant portion of the flame has $|S|>0.25$, and the shape factor distribution is highly skewed negatively. Similar results were reported by Pope et al. [20], Shepherd and Ashurt [21] and Rutland and Trouvé [17]. This skewness indicates that saddle-point structures are substantially more probable than elliptical regions. Moreover, the shape factor shows strong local variablity on the flame surface (not shown). Although the highest probability configuration is cylindrical, the variations in shape factor indicate that, in terms of curvature, the flame structure is not a priori two-dimensional.

\section{Conclusions}

1. It is shown that three dimensional, timedependent simulations of a fully turbulent flame that is anchored on a slot burner are possible using AMR with the DRM-19 chemical mechanism. The slot flame is important because it represents several aspects of practical premixed combustors. In particular, the flame undergoes large stretch rates because the base is anchored and reactants flow directly into the flame.

2. The height of the simulated flame (which is based on the $\bar{c}=0.5$ ) is $41 \mathrm{~mm}$, which in good agreement with the measured value of $40 \mathrm{~mm}$. The parabolic shape of the time-averaged simulated flame is also similar to the experiment. Furthermore, the global consumption speed of the simulated flame (the volume per second of reactants consumed divided by the time-averaged flame area) is in satisfactory agreement with the experiment.

3. The wrinkled shape of the simulated flame has several visible similarities to the experiment: reaction layers are thin (typically thinner than $1 \mathrm{~mm}$ ) and the wavelengths of large wrinkles are 5-10 mm. Wrinkles amplify to become long fingers of reactants that burn through at a neck region, forming perforated flame sheets and isolated pockets of reactants. Thus both the simulated flame and the experiment are in the "corrugated flamelet regime.'

4. The thickness of the computed flame brush increases in the streamwise direction and displays agreement with the experiment.

5. The normalized flame surface density profiles display the same general shapes as the experiment. More quantitative comparisons are difficult at this point because of of extreme sensitivities to slight differences in the development of the flame and the methodology used to compute flame surface density in the experiment which cannot be directly reproduced for the simulation.

6. The degree of agreement between the simulations and the experiment is sufficient to conclude that low Mach number model with the DRM-19 mechanism and AMR correctly simulates several major features of the experiment.

7. The flame surface density was computed using the exact $3 \mathrm{D}$ method as well as the approximate $2 \mathrm{D}$ method that has been used in all experiments to date. Results indicate that the $2 \mathrm{D}$ values differ from the $3 \mathrm{D}$ values by more than $25 \%$, suggesting the need to statistically correct the data when using the 2D method.

8. The simulations indicate the importance of eliminating unwanted shear layers by providing an outer coflow of products at a velocity that is matched to the products produced by the flame. Both the simulation and experiment provided this coflow. Additional simulations not presented show that if the coflow velocity is not properly matched, there is a substantial change in flame height.

9. The work indicates that reasonable agreement is possible if only two parameters are used to quantify the incident turbulence, and these are matched to the experiment: the r.m.s. velocity fluctuation level and 
the longitudinal integral length scale.

Future work will explore higher turbulent intensities and assess whether a detailed match to the power spectrum is required to substantially improve the comparison between experiment and simulation. We will use the resulting suite of simulations to explore in greater detail turbulence chemistry interaction for these types of flames.

\section{Acknowledgments}

Computations were performed at NERSC at LBNL and NCCS at ORNL. The work of J. Bell, M. Day, J. Grcar and M. Lijewski was supported by the DOE Office of Science through the SciDAC program by the Office of Advanced Scientific Computing Research, Mathematical, Information, and Computational Sciences Division.

\section{References}

[1] S. S. Sattler, D. A. Knaus, F. C. Gouldin, Proc. Combust. Inst. 29 (2002) 1785-1795.

[2] I. G. Shepherd, R. K. Cheng, T. Plessing, C. Kortschik, N. Peters, Proc. Combust. Inst. 29 (2002) 1833-1840.

[3] D. Most, F. Dinkelacker, A. Leipertz, Proc. Combust. Inst. 29 (2002) 1801-1806.

[4] Y.-C. Chen, P. A. M. Kalt, R. W. Bilger, N. Swaminathan, Proc. Combust. Inst. (2002) 1863-1871.

[5] N. Peters, Turbulent Combustion, Cambridge University Press, Cambridge, 2000.

[6] L. Vervisch, R. Hauguel, P. Domingo, M. Rullaud, J. Turbulence 5 (2004) 1-36.

[7] J. B. Bell, M. S. Day, I. G. Shepherd, M. Johnson, R. K. Cheng, J. F. Grcar, V. E. Beckner, M. J. Lijewski, Proc. Natl. Acad. Sci. USA 102 (29) (2005) 1000610011.

[8] S. A. Filatyev, J. F. Driscoll, C. D. Carter, J. M. Donbar, Combust. Flame 141 (2005) 1-21.

[9] R. K. Cheng, I. G. Shepherd, Combust. Flame 85 (1991) 7-26.

[10] M. J. Berger, P. Colella, J. Comput. Phys. 82 (1) (1989) 64-84.

[11] A. S. Almgren, J. B. Bell, P. Colella, L. H. Howell, M. L. Welcome, J. Comput. Phys. 142 (1998) 1-46.

[12] M. S. Day, J. B. Bell, Combust. Theory Modelling 4 (2000) 535-556.

[13] J. B. Bell, M. S. Day, J. F. Grcar, Proc. Combust. Inst. 29 (2002) 1987-1993.

[14] M. Frenklach, H. Wang, M. Goldenberg, G. P. Smith, D. M. Golden, C. T. Bowman, R. K. Hanson, W. C. Gardiner, V. Lissianski, GRI-Mech-An Optimized Detailed Chemical Reaction Mechanism for Methane Combustion, Tech. Rep. GRI-95/0058, Gas Research Institute, http: / / www .me.berkeley . edu/gri_mech/(1995).

[15] J. Mueller, J. Driscoll, D. Reuss, M. Drake, M. Rosalik, Combustion and Flame 112 (1998) 342-358.

[16] S. S. Girimaji, S. B. Pope, J. Fluid Mech. 234 (1991) 247-277.

[17] C. J. Rutland, A. Trouvé, Combust. Flame 94 (1993) 41-57.

[18] W. T. Ashurst, I. G. Shepherd, Combust. Sci. Tech. (1997) 115-144
[19] J. F. Hughes, Differential Geometry of Implicit Surfaces in 3-Space - A Primer, Technical Report CS-0305, Department of Computer Sciences, Brown University (2003).

[20] S. B. Pope, P. K. Yeung, S. S. Girimaji, Phys. Fluids A 1 (1989) 2010-2018.

[21] I. G. Shepherd, Proc. Combust. Inst. 24 (1992) 485491. 


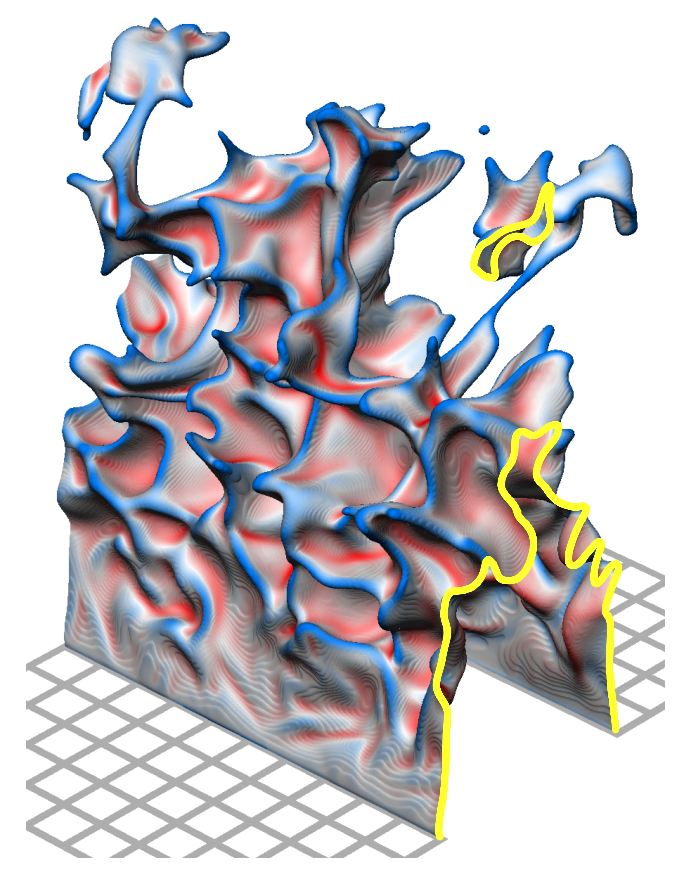

Fig. 1: Simulated instantaneous flame surface represented by the isotherm at $1684 \mathrm{~K}$, which coincides with peak heat release in a simulation of the flat, laminar flame. The flame surface is colored by the mean curvature where blue is negative and red is positive.
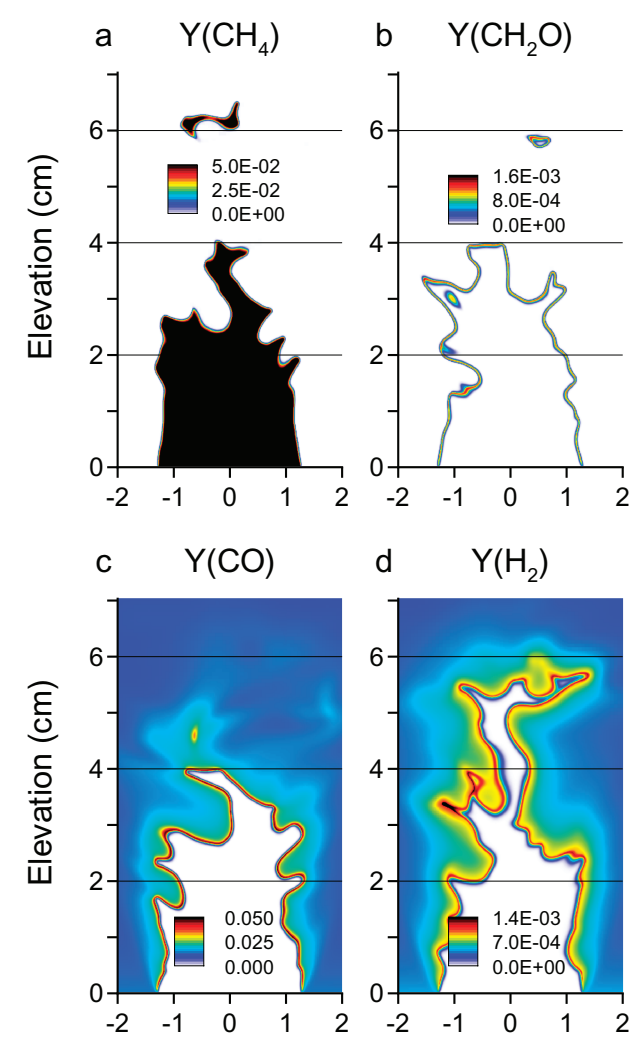

d $\quad \mathrm{Y}\left(\mathrm{H}_{2}\right)$
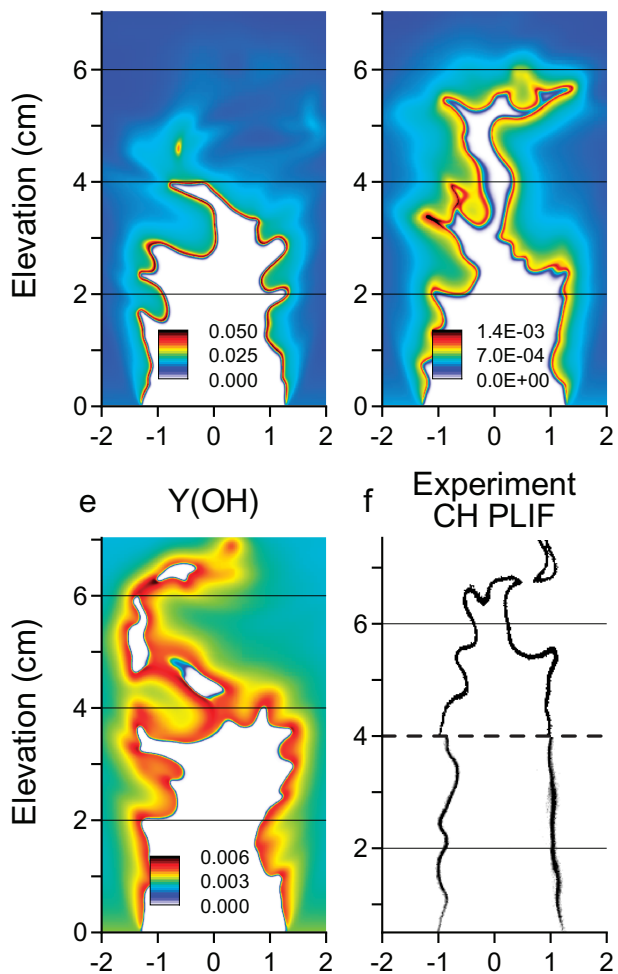

f Experiment

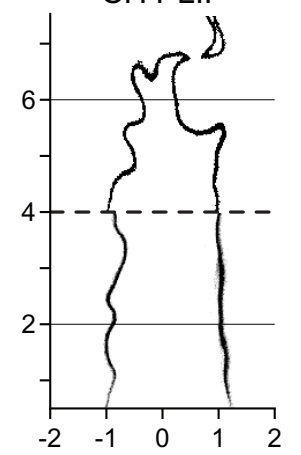

Distance From Slot Centerline $(\mathrm{cm})$

Fig. 2: Structure of the simulated species concentrations (ae) and the measured $\mathrm{CH}$ reaction layers (f). For the images (a-e) taken from the simulation, the slot cross sections are spaced at $0.85 \mathrm{~cm}$ intervals from the front of Fig. 1 whose yellow boundary coincides with (a). Each section displays the mass concentration of a different species in the simulation. 


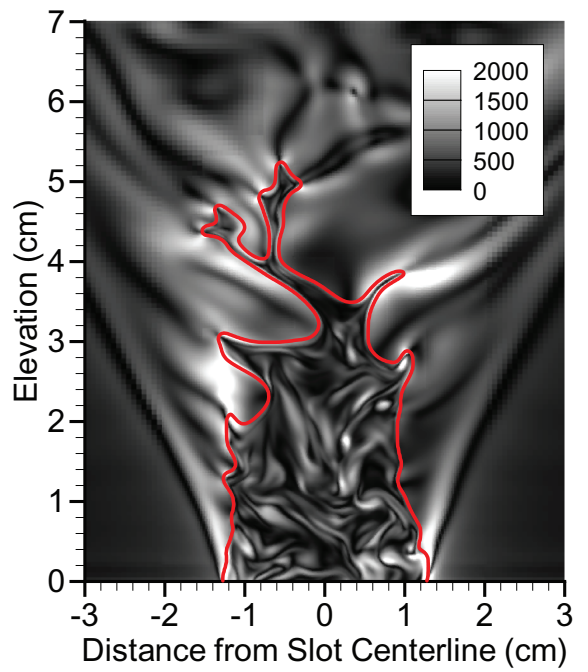

Fig. 3: Vortical cut of the magnitude of vorticity. Light regions are regions of high vorticity. The flame location, defined as the $T=1684 K$ isotherm is red.
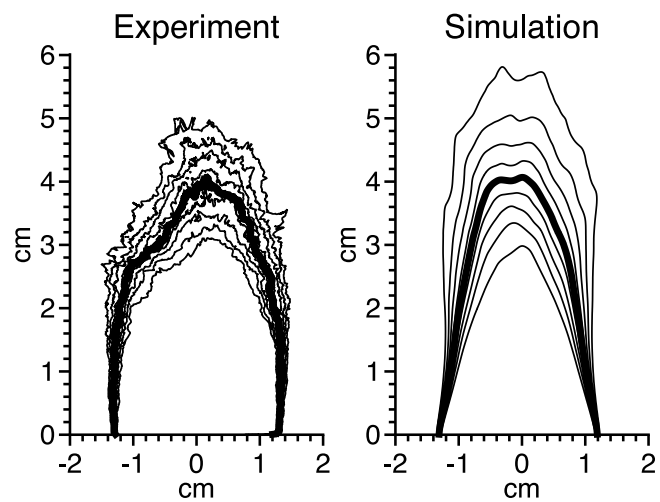

Fig. 4: Flame brush depicted by $0.1-0.9$ contours of (left) averaged Mie scattering images, and (right) temporally and spatially averaged computed temperature field. Thick lines are the 0.5 contours.

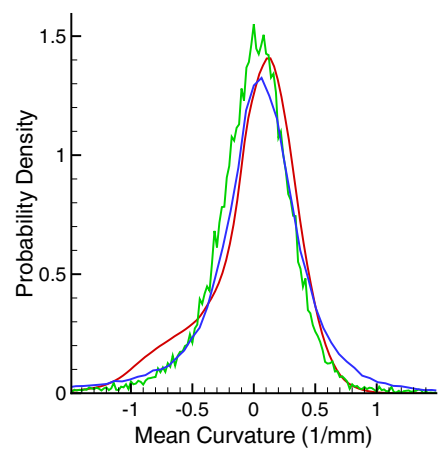

Fig. 6: PDF of Curvature. Green is the experimental data, blue is the two-dimensional curvature computed from the simulation and red is the three-dimensional mean curvature.

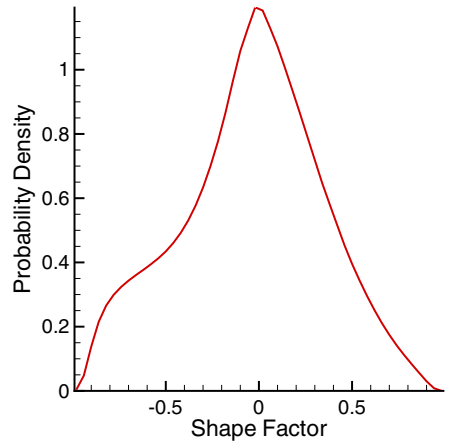

Fig. 7: PDF of shape factor $S$ from the simulation. 
Flame Surface Density, $\Sigma(1 / \mathrm{mm})$

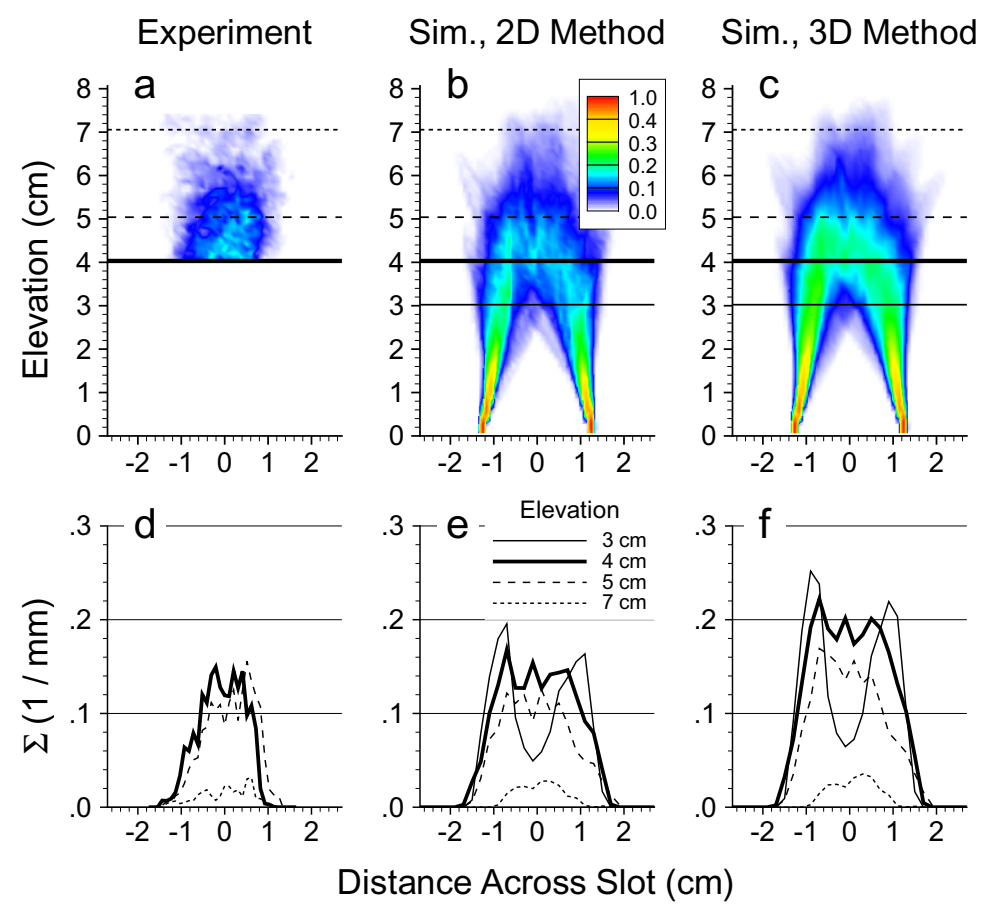

Fig. 5: Flame surface density of the simulation computed by two different methods, (left) 2D and (right) 3D, that are described in the text. Densities are shown both (top, $1 \mathrm{~mm}$ bins) as contours and (bottom, $2 \mathrm{~mm}$ bins) in profiles. 